\title{
DUALITIES FOR MODAL ALGEBRAS FROM THE POINT OF VIEW OF TRIPLES
}

\author{
DIRK HOFMANN AND PEDRO NORA
}

\begin{abstract}
In this paper we show how the theory of monads can be used to deduce in a uniform manner several duality theorems involving categories of relations on one side and categories of algebras with homomorphisms preserving only some operations on the other. Furthermore, we investigate the monoidal structure induced by Cartesian product on the relational side and show that in some cases the corresponding operation on the algebraic side represents bimorphisms.
\end{abstract}

\section{INTRODUCTION}

The title (and content) of this note is clearly inspired by the paper [Negrepontis, 1971] where the author derives the classical duality theorems of Gelfand and Pontrjagin "employing the theory of triples and using only a minimum of analytic information" [Negrepontis, 1971. We recall that Gelfand's duality theorem states that the category $C^{*}$-Alg of commutative and unital $C^{*}$-algebras and homomorphisms is equivalent to the dual of the category CompHaus of compact Hausdorff spaces and continuous maps. The argument of [Negrepontis, 1971] can be summarised as follows:

- the categories $C^{*}$-Alg and CompHaus ${ }^{\text {op }}$ are both tripleable (= monadic) over Set, that is, CompHaus $^{\mathrm{op}}$ is equivalent to the category of Eilenberg-Moore algebras of some triple (= monad), and also $C^{*}$-Alg is equivalent to the category of Eilenberg-Moore algebras of some monad (the proof of the latter fact needs the "minimum of analytic information");

- the induced monads are equal.

At the heart of the equivalence between Kripke semantics and algebraic semantics in modal logic lies Jónnson and Tarski's representation theorem for Boolean algebras with an operator (see Jónsson and Tarski, 1951, 1952]), which eventually led to a duality between the category of Boolean algebras with an operator and homomorphisms and the category of "Stone Kripke frames". The latter category is elegantly described in [Kupke et al., 2004] as the category of coalgebras for the Vietoris functor on the category Stone of Stone spaces and continuous maps. As advocated in Halmos, 1956 (see also Wright, 1957]) and later in [Sambin and Vaccaro, 1988], this duality result can be seen as a consequence of the more general duality between the category StoneRel of Stone spaces and Boolean relations and the category of Boolean algebras with "hemimorphisms", that is, maps preserving finite suprema but not necessarily finite infima.

The starting point of this paper is the observation that the Vietoris functor is actually part of a monad on Stone, and StoneRel is equivalent to the Kleisli category of this monad. This fact opens the door to use an argumentation similar to the one in Negrepontis, 1971, but now with the Kleisli construction in lieu of the Eilenberg-Moore construction. We hasten to remark that the situation here is actually much simpler than the one described above, basically because it is usually easy the see that a category is equivalent to a Kleisli category whereby monadicity is

Date: July 17, 2014.

2010 Mathematics Subject Classification. 03G05, 03G10, 18A40, 18C15, 18C20, 54H10 .

Key words and phrases. Boolean algebra, distributive lattice, monad, Kleisli construction, dual equivalence, Stone space, spectral space, Vietoris functor, tensor product.

Partial financial assistance by Portuguese funds through CIDMA (Center for Research and Development in Mathematics and Applications), and the Portuguese Foundation for Science and Technology ("FCT - Fundação para a Ciência e a Tecnologia"), within the project PEst-OE/MAT/UI4106/2014, and by the project NASONI under the contract PTDC/EEI-CTP/2341/2012 is gratefully acknowledged. 
a property much harder to establish. Therefore, using the theory of monads, in this paper we derive in a uniform way several duality theorems involving categories of relations and categories of algebras with "hemimorphisms". Our examples include Halmos duality as well as a similar result for the category SpecRel of spectral spaces and spectral relations (respectively Priestley spaces and Priestley relations, see [Cignoli et al. 1991]). We then proceed by investigating the monoidal structure on SpecRel and StoneRel induced by the topological product of spaces, and show that this structure corresponds under the aforementioned dualities to a tensor product which represents bimorphisms.

\section{Kleisli adjunctions}

The purpose of this section is to recall briefly some well-known results about monads and adjunctions. The main focus lies on the fact that the principal constructions are functorial and give rise to (large) adjunctions as explained in [Pumplün, 1970, 1988] and [Tholen, 1974] (see also Porst, 1994). For more information on monads we refer to MacDonald and Sobral, 2004.

Definition 1.1. An adjunction $(F \dashv G, \eta, \varepsilon): \mathrm{A} \rightleftarrows \mathrm{X}$ consists of

- the right adjoint functor $G: \mathrm{A} \rightarrow \mathrm{X}$,

- the left adjoint functor $F: \mathrm{X} \rightarrow \mathrm{A}$,

- the unit natural transformation $\eta: 1_{\mathrm{X}} \rightarrow G F$, and

- the co-unit natural transformation $\varepsilon: F G \rightarrow 1_{\mathrm{A}}$

such that the diagrams
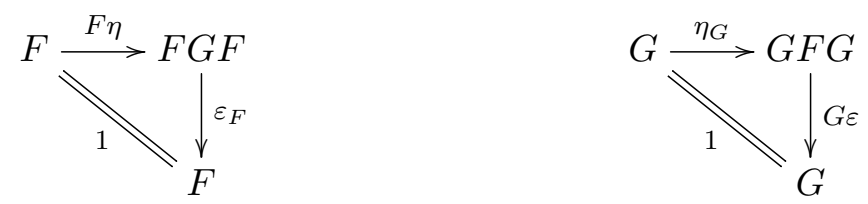

commute. Let $(F \dashv G, \eta, \varepsilon): \mathrm{A} \rightleftarrows \mathrm{X}$ and $\left(F^{\prime} \dashv G^{\prime}, \eta^{\prime}, \varepsilon^{\prime}\right): \mathrm{A}^{\prime} \rightleftarrows \mathrm{X}$ be adjunctions over a fixed base category $\mathrm{X}$. A right morphism of adjunctions over $\mathrm{X}$ is a functor $J: \mathrm{A} \rightarrow \mathrm{A}^{\prime}$ with $G=G^{\prime} J$. Likewise, a left morphism of adjunctions over $\mathrm{X}$ is a functor $J: \mathrm{A} \rightarrow \mathrm{A}^{\prime}$ with $F^{\prime}=J F$.

We denote by $\operatorname{RAdj}(X)$ the category of adjunctions over $X$ and right morphisms of adjunctions over $\mathbf{X}$, and $\operatorname{LAdj}(X)$ denotes the category of adjunctions over $X$ and left morphisms of adjunctions over $\mathrm{X}$.

Remark 1.2. Note that we do not require $F^{\prime}=J F$ in the definition of a right morphism of adjunctions; however, there is a canonical natural transformation $\kappa: F^{\prime} \rightarrow J F$ defined as the composite

$$
F^{\prime} \stackrel{F^{\prime} \eta}{\longrightarrow} F^{\prime} G F=F^{\prime} G^{\prime} J F \stackrel{\varepsilon_{J F}}{\longrightarrow} J F,
$$

and for a left morphism of adjunctions $J$ we have a canonical natural transformation $\iota: G \rightarrow G^{\prime} J$ defined as the composite

$$
G \stackrel{\eta_{G}^{\prime}}{\longrightarrow} G^{\prime} F^{\prime} G=G^{\prime} J F G \stackrel{G^{\prime} J \varepsilon}{\longrightarrow} G^{\prime} J
$$

Remark 1.3. An adjunction is an equivalence whenever both the unit and the co-unit are natural isomorphisms. Every adjunction $(F \dashv G, \eta, \varepsilon): \mathrm{A} \rightleftarrows \mathrm{X}$ induces an equivalence between the (possibly empty) full subcategories

$\operatorname{Fix}(\mathrm{X})=\left\{X \in \mathrm{X} \mid \eta_{X}\right.$ is an isomorphism $\}$ and $\operatorname{Fix}(\mathrm{A})=\left\{A \in \mathrm{A} \mid \varepsilon_{A}\right.$ is an isomorphism $\}$.

Definition 1.4. A monad $\mathbb{T}=(T, e, m)$ on a category $\mathbf{X}$ consists of a functor $T: X \rightarrow \mathbf{X}$ together with natural transformations $e: 1_{\mathbf{X}} \rightarrow T$ (unit) and $m: T T \rightarrow T$ (multiplication) such 
that the diagrams
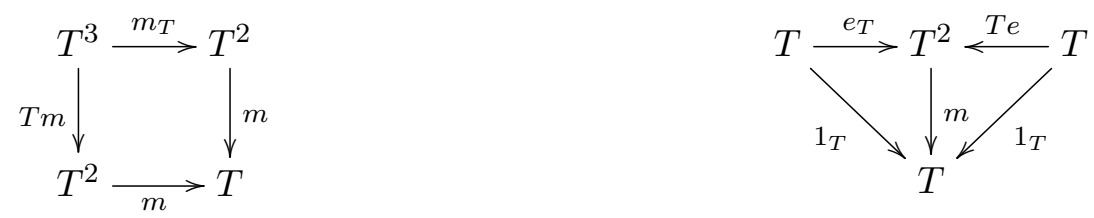

commute. For monads $\mathbb{T}, \mathbb{T}^{\prime}$ on a category $X$, a monad morphism $j: \mathbb{T} \rightarrow \mathbb{T}^{\prime}$ is a natural transformation $j: T \rightarrow T^{\prime}$ such that the diagrams
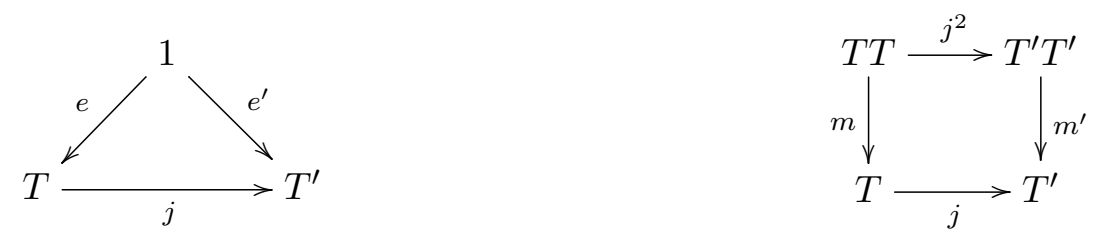

commute, where $j^{2}=j_{T^{\prime}} \cdot T j=T^{\prime} j \cdot j_{T}$.

The category of monads on $\mathrm{X}$ and monad morphisms is denoted by Mon $(\mathrm{X})$.

Examples 1.5. (1) The identity monad $\mathbb{1}=(1,1,1)$. Trivially, the identity functor $1: \mathrm{X} \rightarrow \mathrm{X}$ together with the identity transformation $1: 1 \rightarrow 1$ forms a monad. It is the initial monad: for every monad $\mathbb{T}=(T, e, m)$ on $\mathbf{X}$, the unit $e$ is the unique monad morphism $\mathbb{1} \rightarrow \mathbb{T}$.

(2) The powerset monad $\mathbb{P}=(P, e, m)$ on Set. The powerset functor $P$ : Set $\rightarrow$ Set sends each set to its powerset $P X$ and each function $f: X \rightarrow Y$ to the direct image function $P f: P X \rightarrow P Y, A \mapsto f[A]$. The $X$-component of the natural transformation $e$ respectively $m$ is given by "taking singletons" $e_{X}: X \rightarrow P X, x \mapsto\{x\}$ and union $m_{X}: P P X \rightarrow P X, \mathcal{A} \mapsto \bigcup \mathcal{A}$.

(3) The filter monad $\mathbb{F}=(F, e, m)$ on Set. The filter functor $F:$ Set $\rightarrow$ Set sends a set $X$ to the set $F X$ of all filters on $X$ and, for $f: X \rightarrow Y$, the map $F f$ sends a filter $\mathfrak{f}$ on $X$ to the filter $\left\{B \subseteq Y \mid f^{-1}[B] \in \mathfrak{f}\right\}$ on $Y$. The natural transformations $e: 1 \rightarrow F$ and $m: F F \rightarrow F$ are given by

$$
e_{X}(x)=\dot{x}=\{A \subseteq X \mid x \in A\} \quad \text { and } \quad m_{X}(\mathfrak{F})=\left\{A \subseteq X \mid A^{\#} \in \mathfrak{F}\right\},
$$

for all sets $X, \mathfrak{F} \in F F X$ and $x \in X$, where $A^{\#}=\{\mathfrak{f} \in F X \mid A \in \mathfrak{f}\}$.

(4) The filter monad $\mathbb{F}=\left(F_{\tau}, e, m\right)$ on Top. For a topological space $X, F_{\tau} X$ is the set of all filters on the lattices of opens of $X$, equipped with the topology generated by the sets $U^{\#}$, for $U \subseteq X$ open. The continuous map $F_{\tau} f: F_{\tau} X \rightarrow F_{\tau} Y$, for $f: X \rightarrow Y$ in Top, and the unit and the multiplication are defined as above. For more information we refer to Escardó, 1997.

In Section 2 we will describe topological counterparts of the powerset monad.

In the remainder of this section we will construct adjunctions

$$
\operatorname{Mon}(X)^{\text {op }} \rightleftarrows \operatorname{RAdj}(X) \quad \text { and } \quad \operatorname{LAdj}(X) \rightleftarrows \operatorname{Mon}(X)
$$

and identify their fixed objects. We explain how these results can be used to establish equivalences of categories.

Every adjunction $(F \dashv G, \eta, \varepsilon): \mathbf{A} \rightleftarrows \mathbf{X}$ over $\mathbf{X}$ induces a monad $\mathbb{T}=(T, e, m)$ on $\mathbf{X}$ defined by

$$
T=G F, \quad \quad e=\eta \quad \text { and } \quad m=G \varepsilon_{F} .
$$

Moreover, every right morphism $J$ of adjunctions $(F \dashv G, \eta, \varepsilon): \mathrm{A} \rightleftarrows \mathrm{X}$ and $\left(F^{\prime} \dashv G^{\prime}, \eta^{\prime}, \varepsilon^{\prime}\right)$ : $\mathrm{A}^{\prime} \rightleftarrows \mathrm{X}$ over $\mathrm{X}$ induces a monad morphism

$$
j=G^{\prime} \kappa: \mathbb{T}^{\prime} \rightarrow \mathbb{T}
$$


between the induced monads. These constructions define the object and the morphism part of the functor $M^{\mathrm{X}}: \operatorname{RAdj}(\mathrm{X}) \rightarrow \operatorname{Mon}(\mathrm{X})^{\mathrm{op}}$. Likewise, every left morphism $J$ of adjunctions induces a monad morphism

$$
j=\iota_{F}: \mathbb{T} \rightarrow \mathbb{T}^{\prime}
$$

and we obtain a functor $M_{\mathrm{X}}: \operatorname{LAdj}(\mathrm{X}) \rightarrow \operatorname{Mon}(\mathrm{X})$. As we show next, both functors have adjoints given by well-known constructions.

Definition 1.6. Let $\mathbb{T}=(T, e, m)$ be a monad on a category $\mathrm{X}$. A $\mathbb{T}$-algebra (also called Eilenberg-Moore algebra) is a pair $(X, \alpha)$ consisting of an $\mathrm{X}$-object $X$ and an $\mathrm{X}$-morphism $\alpha: T X \rightarrow X$ making the diagrams
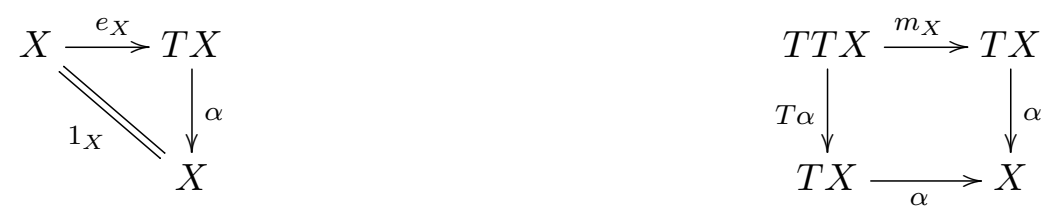

commute. Let $(X, \alpha)$ and $(Y, \beta)$ be $\mathbb{T}$-algebras. A map $f: X \rightarrow Y$ is a $\mathbb{T}$-algebra homomorphism if the diagram

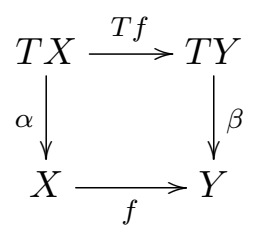

commutes.

The category of $\mathbb{T}$-algebras and $\mathbb{T}$-algebra homomorphisms is denoted by $X^{\mathbb{T}}$. There is a canonical forgetful functor $G^{\mathbb{T}}: \mathrm{X}^{\mathbb{T}} \rightarrow \mathrm{X},(X, \alpha) \mapsto X$ with left adjoint $F^{\mathbb{T}}: \mathrm{X} \rightarrow \mathrm{X}^{\mathbb{T}}, X \mapsto$ $\left(T X, m_{X}\right)$. Moreover, every monad morphism $j: \mathbb{T} \rightarrow \mathbb{T}^{\prime}$ induces a functor

$$
\mathrm{X}^{j}: \mathrm{X}^{\mathbb{T}^{\prime}} \rightarrow \mathrm{X}^{\mathbb{T}},(X, \alpha) \mapsto\left(X, \alpha \cdot j_{X}\right)
$$

with $G^{\mathbb{T}} X^{j}=G^{\mathbb{T}^{\prime}}$, that is, $X^{j}:\left(F^{\mathbb{T}^{\prime}} \dashv G^{\mathbb{T}^{\prime}}\right) \rightarrow\left(F^{\mathbb{T}} \dashv G^{\mathbb{T}}\right)$ is a right morphism of adjunctions over $\mathrm{X}$. These constructions define indeed a functor

$$
X^{(-)}: \operatorname{Mon}(X)^{\text {op }} \rightarrow \operatorname{RAdj}(X) .
$$

It is easy to see that $F^{\mathbb{T}} \dashv G^{\mathbb{T}}$ induces $\mathbb{T}$, that is, $\mathbb{T}=M^{\mathrm{X}} \cdot \mathrm{X}^{(-)}(\mathbb{T})$. On the other hand, for every adjunction $(F \dashv G, \eta, \varepsilon): \mathrm{A} \rightleftarrows \mathrm{X}$ over $\mathbf{X}$ (with induced monad $\mathbb{T}$ ) we have a canonical comparison functor $K: \mathrm{A} \rightarrow \mathrm{X}^{\mathbb{T}}$ defined by $K(A)=\left(G A, G \varepsilon_{A}\right)$ and $K f=G f$; hence,

$$
K:((F \dashv G, \eta, \varepsilon): \mathrm{A} \rightleftarrows \mathrm{X}) \rightarrow\left(\left(F^{\mathbb{T}} \dashv G^{\mathbb{T}}, \eta, \varepsilon\right): \mathrm{X}^{\mathbb{T}} \rightleftarrows \mathrm{X}\right)
$$

is a right morphism of adjunctions over $\mathrm{X}$. For a right morphism $J$ of adjunctions $(F \dashv G, \eta, \varepsilon)$ : $\mathrm{A} \rightleftarrows \mathrm{X}$ and $\left(F^{\prime} \dashv G^{\prime}, \eta^{\prime}, \varepsilon^{\prime}\right): \mathrm{A}^{\prime} \rightleftarrows \mathrm{X}$ over $\mathrm{X}$, the diagram

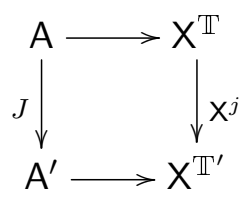

commutes, that is, the family of all comparison functors defines a natural transformation $1 \rightarrow$ $\mathrm{X}^{(-)} M^{\mathrm{X}}$. In fact, this transformation together with the family $\left(\mathbb{T}=M^{\mathrm{X}} \mathbf{X}^{(-)}(\mathbb{T})\right)_{\mathbb{T}}$ are the units of the adjunction

$$
M^{\mathrm{X}} \dashv \mathrm{X}^{(-)}: \operatorname{Mon}(\mathrm{X})^{\mathrm{op}} \rightleftarrows \operatorname{RAdj}(\mathrm{X}) \text {. }
$$

Clearly, $\operatorname{Fix}(\operatorname{Mon}(X))=\operatorname{Mon}(X)$; but, deviating slightly from Remark 1.3 , we let the fixed subcategory Fix $(\operatorname{RAdj}(X))$ consist of those objects whose component of the unit is an equivalence of categories, rather than an isomorphism. An object of $\operatorname{Fix}(\operatorname{RAdj}(X))$ is called a monadic adjunction, these adjunctions are characterised by the following 
Theorem 1.7 ([Beck, 1967]). An adjunction $(F \dashv G, \eta, \varepsilon): \mathrm{A} \rightleftarrows \mathrm{X}$ is monadic if and only if $G$ reflects isomorphisms and $\mathrm{A}$ has and $G$ preserves all $G$-contractible coequaliser pairs.

As we will not make use of monadic adjunctions in the sequel, we refer to MacDonald and Sobral, 2004 for a proof and also for the definition of the pertinent notions.

Example 1.8. The canonical forgetful functor $|-|:$ CompHaus $\rightarrow$ Set from the category of compact Hausdorff spaces and continuous maps has a left adjoint given by Čech-Stone compactification, and it is shown in Manes, 1969 that this adjunction is monadic. The induced monad on Set is the ultrafilter monad.

The "equivalence" between monads and monadic adjunctions provides a general principle to prove equivalence of two categories: firstly, show that both categories are part of monadic adjunctions over the same category $\mathbf{X}$; and secondly, show that these adjunctions induce isomorphic monads. This idea was used in Negrepontis, 1971 to obtain the classical duality theorems of Gelfand and Pontrjagin.

We will explain now how $M_{\mathrm{X}}: \operatorname{LAdj}(\mathrm{X}) \rightarrow \operatorname{Mon}(\mathrm{X})$ is part of an adjunction.

Definition 1.9. Let $\mathbb{T}=(T, e, m)$ be a monad over $X$. The Kleisli category $X_{\mathbb{T}}$ has the same objects as $\mathrm{X}$, and a morphism $f: X \longrightarrow Y$ in $\mathrm{X}_{\mathbb{T}}$ is an $\mathrm{X}$-morphism $f: X \rightarrow T Y$. Given morphisms $f: X \longrightarrow Y$ and $g: Y \longrightarrow Z$ in $\mathrm{X}_{\mathbb{T}}$, the composite $g \cdot f: X \longrightarrow Z$ is defined as

$$
X \stackrel{f}{\longrightarrow} T Y \stackrel{T g}{\longrightarrow} T T Z \stackrel{m_{Z}}{\longrightarrow} T Z .
$$

Then $e_{X}: X \rightarrow T X$ is the identity on $X$ in $\mathrm{X}_{\mathbb{T}}$.

We have a canonical adjunction $F_{\mathbb{T}} \dashv G_{\mathbb{T}}: \mathrm{X}_{\mathbb{T}} \rightleftarrows \mathrm{X}$, where

$$
G_{\mathbb{T}}: \mathbf{X}_{\mathbb{T}} \rightarrow \mathbf{X}, f: X \longrightarrow Y \mapsto T X \stackrel{T f}{\longrightarrow} T T Y \stackrel{m_{Y}}{\longrightarrow} T Y
$$

and

$$
F_{\mathbb{T}}: \mathrm{X} \rightarrow \mathrm{X}_{\mathbb{T}}, \quad f: X \rightarrow Y \mapsto X \stackrel{f}{\rightarrow} Y \stackrel{e_{Y}}{\rightarrow} T Y .
$$

Example 1.10. For the powerset monad $\mathbb{P}$ on Set, the category $\operatorname{Set}_{\mathbb{P}}$ is equivalent to the category Rel of sets and relations by interpreting a map $f: X \rightarrow P Y$ as a relation $X \rightarrow Y$ from $X$ to $Y$. Under this equivalence, $F_{\mathbb{P}}:$ Set $\rightarrow$ Set $\mathbb{P}$ corresponds to the inclusion functor Set $\rightarrow$ Rel and $G_{\mathbb{P}}: \operatorname{Set}_{\mathbb{P}} \rightarrow$ Set corresponds to the functor Rel $\rightarrow$ Set which sends a set $X$ to its powerset $P X$ and a relation $r: X \rightarrow Y$ to the map $P X \rightarrow P Y, A \mapsto r[A]$.

Every monad morphism $j: \mathbb{T} \rightarrow \mathbb{T}^{\prime}$ induces a functor $\mathbf{X}_{j}: \mathbf{X}_{\mathbb{T}} \rightarrow \mathbf{X}_{\mathbb{T}^{\prime}}$ which acts as the identity on objects and sends $f: X \longrightarrow Y$ to $X \stackrel{f}{\rightarrow} T Y \stackrel{j_{Y}}{\longrightarrow} T^{\prime} Y$. One clearly has $F_{\mathbb{T}^{\prime}}=\mathrm{X}_{j} F_{\mathbb{T}}$, hence $\mathrm{X}_{j}$ is a left morphism of adjunctions and we obtain a functor

$$
X_{(-)}: \operatorname{Mon}(X) \rightarrow \operatorname{LAdj}(X) .
$$

As before, the induced monad of $F_{\mathbb{T}} \dashv G_{\mathbb{T}}$ is $\mathbb{T}$ again, that is, $\mathbb{T}=M_{\mathbf{X}} \cdot \mathrm{X}_{(-)}(\mathbb{T})$. For every adjunction $(F \dashv G, \eta, \varepsilon): \mathrm{A} \rightleftarrows \mathrm{X}$ over $\mathrm{X}$ (with induced monad $\mathbb{T}$ ) we have a canonical comparison functor $C: \mathrm{X}_{\mathbb{T}} \rightarrow \mathrm{A}$ sending an object $X$ in $\mathrm{X}_{\mathbb{T}}$ to $F X$ and a morphism $f: X \longrightarrow Y$ to $F X \stackrel{F f}{\longrightarrow} F T Y=F G F Y \stackrel{\varepsilon_{F Y}}{\longrightarrow} F Y$. Since $F=C F_{\mathbb{T}}, C$ is a left morphism of adjunctions. For a left morphism $J$ of adjunctions $(F \dashv G, \eta, \varepsilon): \mathrm{A} \rightleftarrows \mathrm{X}$ and $\left(F^{\prime} \dashv G^{\prime}, \eta^{\prime}, \varepsilon^{\prime}\right): \mathrm{A}^{\prime} \rightleftarrows \mathrm{X}$ over $\mathrm{X}$, the diagram

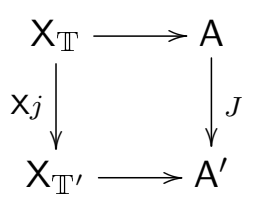


commutes. Therefore $C$ is the $((F \dashv G, \eta, \varepsilon): \mathrm{A} \rightleftarrows \mathrm{X})$-component of a natural transformation $\mathrm{X}_{(-)} M_{\mathrm{X}} \rightarrow 1$, in fact, this transformation is the co-unit of the adjunction

$$
\mathrm{X}_{(-)} \dashv M_{\mathrm{X}}: \operatorname{LAdj}(\mathrm{X}) \rightleftarrows \operatorname{Mon}(\mathrm{X}),
$$

where the unit $1 \rightarrow M_{\mathrm{X}} \mathrm{X}_{(-)}$is given by $\left(\mathbb{T}=M_{\mathrm{X}} \mathrm{X}_{(-)}(\mathbb{T})\right)_{\mathbb{T}}$. The comparison functor $C: \mathrm{X}_{\mathbb{T}} \rightarrow \mathrm{A}$ is always fully faithful, and we call an adjunction $(F \dashv G, \eta, \varepsilon): \mathrm{A} \rightleftarrows \mathrm{X}$ a Kleisli adjunction whenever $C$ is an equivalence. Unlike the situation for monadic adjunctions, Kleisli adjunctions can be easily characterised.

Theorem 1.11. An adjunction $(F \dashv G, \eta, \varepsilon): \mathrm{A} \rightleftarrows \mathrm{X}$ is a Kleisli adjunction if and only if $F$ is essentially surjective on objects.

As for monadic adjunctions, (1) gives a simple scheme to obtain an equivalence between categories $\mathrm{A}$ and $\mathrm{A}^{\prime}$ :

Theorem 1.12. A functor $J: \mathrm{A} \rightarrow \mathrm{A}^{\prime}$ between Kleisli adjunctions $(F \dashv G, \eta, \varepsilon): \mathrm{A} \rightleftarrows \mathrm{X}$ and $\left(F^{\prime} \dashv G^{\prime}, \eta^{\prime}, \varepsilon^{\prime}\right): \mathrm{A}^{\prime} \rightleftarrows \mathrm{X}$ is an equivalence provided that $F^{\prime}=J F$ and the morphism $M_{\mathrm{X}}(J)$ between the induced monads is a natural isomorphism.

We will illustrate this principle with various examples in Section 3

\section{Stably compact spaces, spectral spaces, and Stone spaces}

Our main examples in the following sections involve Stone spaces and spectral spaces as well as the (lower) Vietoris monad. These classes of topological spaces were introduced by M.H. Stone in [Stone, 1936, 1938] in order to give representation theorems for Boolean algebras and for distributive lattices. As we recall below, both are examples of stably compact topological spaces, and stably compact spaces can be equivalently described as compact Hausdorff spaces equipped with a compatible order relation. We stress that, in order to investigate their properties, it is quite useful to pass freely from one description to the other.

In this section we collect a couple of (categorical) properties of stably compact spaces. We will be particularly interested in descriptions of initial structures as they feature in the definition of Stone space and spectral space and play an important role in duality theory (see Theorem 3.1 in the next section). Furthermore, we present properties of the lower Vietoris monad which will be used in the next sections. For more information on these types of spaces we refer to Nachbin, 1950], Gierz et al. 1980], [Jung, 2004], [Tholen, 2009] and [Lawson, 2011]. There exists a vast literature on the (lower) Vietoris construction, see [Vietoris, 1922], Poppe, 1966, Clementino and Tholen, 1997] and Kupke et al., 2004], for instance. A study of stably compact spaces and the Vietoris monad in a more general context can be found in Hofmann, 2014].

We start by recalling some categorical concepts. A source in category A is a family $\left(f_{i}\right.$ : $\left.A \rightarrow A_{i}\right)_{i \in I}$ of morphisms with a common domain $A$. Given a functor $U: \mathrm{A} \rightarrow \mathrm{X}$, a source $\left(f_{i}: A \rightarrow A_{i}\right)_{i \in I}$ in $\mathrm{A}$ is called $U$-initial whenever, for every source $\left(g_{i}: B \rightarrow A_{i}\right)_{i \in I}$ in $\mathrm{A}$ and every morphism $h: U B \rightarrow U A$ in $\mathrm{X}$ with $U f_{i} \cdot h=U g_{i}$ for all $i \in I$, there is a unique $\bar{h}: B \rightarrow A$ in A with $U \bar{h}=h$ and $f_{i} \cdot \bar{h}=g_{i}$ for all $i \in I$. A functor $U: \mathrm{A} \rightarrow \mathrm{X}$ is topological whenever every source $\left(f_{i}: X \rightarrow U A_{i}\right)_{i \in I}$ in $\mathbf{X}$ admits a $U$-initial lifting, that is, a $U$-initial source $\left(\bar{f}_{i}: A \rightarrow A_{i}\right)_{i \in I}$ with $U A=X$ and $U \bar{f}_{i}=f_{i}$ for all $i \in I$. The paradigmatic example of a topological functor is the forgetful functor $|-|:$ Top $\rightarrow$ Set; here the initial lift of a source $f_{i}: X \rightarrow X_{i}$ in Set (where $\left(X_{i}, \tau_{i}\right)$ are topological spaces) is obtain by equipping $X$ with the topology $\tau$ generated by the sets

$$
\left\{f_{i}^{-1}(W) \mid W \in \tau_{i}\right\} \quad(i \in I) .
$$

Finally, a functor $U: \mathrm{A} \rightarrow \mathrm{X}$ is mono-topological whenever every mono-source $\left(f_{i}: X \rightarrow\right.$ $\left.U A_{i}\right)_{i \in I}$ in $\mathrm{X}$ admits a $U$-initial lifting. For instance, with Top $_{0}$ denoting the category of $\mathrm{T}_{0}$ topological spaces and continuous maps, the canonical forgetful functor Top $_{0} \rightarrow$ Set is not 
topological but it is mono-topological. An extensive presentation of the theory of topological functors can be found in Adámek et al., 1990].

Definition 2.1. An ordered compact Hausdorff space (see [Nachbin, 1950]) is a triple $(X, \leq, \tau)$ where $(X, \leq)$ is an ordered set and $\tau$ is a compact Hausdorff topology on $X$ so that $\{(x, y) \mid x \leq y\}$ is closed in $X \times X$.

We follow here [Tholen, 2009 and do not assume the order relation $\leq$ on $X$ to be antisymmetric. In the sequel we write OrdCompHaus for the category of ordered compact Hausdorff spaces and maps preserving both the order and the topology, and denote its full subcategory defined by the objects with anti-symmetric order as OrdCompHaus sep $_{\text {. }}$

As shown in Tholen, 2009, OrdCompHaus is the Eilenberg-Moore category for the ultrafilter monad on the category Ord of (not necessarily anti-symmetric) ordered sets and monotone maps. Therefore OrdCompHaus is complete and the forgetful functor $|-|:$ OrdCompHaus $\rightarrow$ Ord preserves limits. The canonical functor $|-|:$ OrdCompHaus $\rightarrow$ CompHaus is topological, and therefore OrdCompHaus is also cocomplete and the forgetful functor $|-|$ : OrdCompHaus $\rightarrow$ CompHaus has a fully faithful right adjoint and a fully faithful left adjoint CompHaus $\hookrightarrow$ OrdCompHaus; the latter equips a compact Hausdorff space with the discrete order. This functor clearly corestricts to CompHaus $\hookrightarrow$ OrdCompHaus $_{\text {sep }}$ and this corestriction is left adjoint to the forgetful functor $|-|:$ OrdCompHaus $_{\text {sep }} \rightarrow$ CompHaus. Since OrdCompHaus $_{\text {sep }}$ is closed under mono-sources (= point-separating sources) in OrdCompHaus, Theorems 16.8 and 21.40 of Adámek et al., 1990 (the numbering refers to the reprint) imply at once:

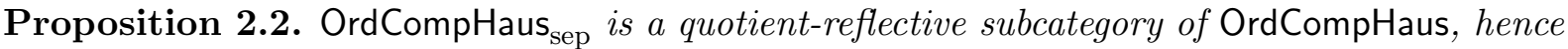
it is complete and cocomplete with limits formed as in OrdCompHaus. The forgetful functor $|-|$ : OrdCompHaus sep $_{\rightarrow} \rightarrow$ CompHaus is mono-topological and therefore every source in OrdCompHaus $_{\text {sep }}$ factors as a surjective map followed by an initial mono-source (with respect to $|-|:$ OrdCompHaus $_{\text {sep }} \rightarrow$ CompHaus).

For a topological space $X$, we consider its underlying order defined by $x \leq y$ whenever the principal filter $\dot{x}$ converges to $y$, or, equivalently, whenever $y \in \overline{\{x\}}$. Clearly, every continuous map is monotone with respect to this order. We also note that this order relation is dual to the specialisation order. For continuous maps $f: X \rightarrow Y$ and $g: Y \rightarrow X$, we say that $f$ is left adjoint to $g$, written as $f \dashv g$, if $x \leq g(f(x))$ and $f(g(y)) \leq y$, for all $x \in X$ and $y \in Y$. Given $f$, there exists up to equivalence at most one such $g$, and in this case we call $f$ a left adjoint continuous map.

Given an ordered compact Hausdorff space $(X, \leq, \tau)$, one defines a new topology $\downarrow \tau$ on $X$ which contains precisely those elements of $\tau$ which are down-closed; and this construction defines a functor $K:$ OrdCompHaus $\rightarrow$ Top. Note that the underlying order of $K X$ is precisely the order relation of $X$. We obtain a commuting diagram of functors

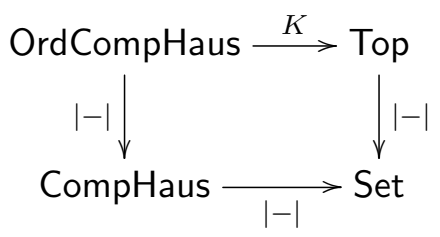

where both vertical arrows represent topological functors and both horizontal arrows monadic functors (see Example 1.8 and [Simmons, 1982], the latter only considers anti-symmetric orders; however, the general case is similar).

Proposition 2.3. Let $\left(f_{i}: X \rightarrow X_{i}\right)_{i \in I}$ be a source in OrdCompHaus. Then $\left(f_{i}: X \rightarrow X_{i}\right)_{i \in I}$ is initial with respect to $|-|:$ OrdCompHaus $\rightarrow$ CompHaus if and only if $\left(K f_{i}: K X \rightarrow K X_{i}\right)_{i \in I}$ is initial with respect to $|-|:$ Top $\rightarrow$ Set.

Proof. This follows from the description of the initial lifts of [Tholen, 2009, Proposition 3]. 
Definition 2.4. A topological space $X$ is called stably compact whenever $X$ is a locally compact, sober, and the compact down-closed subsets are closed under finite intersections. A continuous map $f: X \rightarrow Y$ between stably compact spaces is spectral map whenever $f^{-1}(A)$ is compact for every compact down-closed subset $A \subseteq Y$.

A useful criterion for verifying if a continuous map is spectral is given by the fact that every left adjoint continuous map between stably compact spaces is spectral. The category of stably compact spaces and spectral maps is denoted as StablyComp.

Theorem 2.5. The functor $K:$ OrdCompHaus $\rightarrow$ Top restricts to an isomorphism

$$
K: \text { OrdCompHaus }_{\text {sep }} \rightarrow \text { StablyComp }
$$

which commutes with the respective forgetful functors to Set. Hence, StablyComp is complete and cocomplete and the inclusion functor StablyComp $\rightarrow$ Top preserves limits.

Proof. See Jung, 2004, for instance.

Via this equivalence, $|-|$ : OrdCompHaus sep $\rightarrow$ CompHaus corresponds to a mono-topological faithful functor $|-|$ : StablyComp $\rightarrow$ CompHaus with fully faithful left adjoint CompHaus $\hookrightarrow$ StablyComp which is just the inclusion functor. The next result affirms that initial liftings of mono-sources in StablyComp can be obtained as in Top.

Proposition 2.6. For a mono-source $\left(f_{i}: X \rightarrow X_{i}\right)_{i \in I}$ in StablyComp, the following assertions are equivalent.

(i) $\left(f_{i}: X \rightarrow X_{i}\right)_{i \in I}$ is initial with respect to $|-|$ : StablyComp $\rightarrow$ CompHaus.

(ii) $\left(f_{i}: X \rightarrow X_{i}\right)_{i \in I}$ is initial with respect to $|-|:$ StablyComp $\rightarrow$ Set.

(iii) $\left(f_{i}: X \rightarrow X_{i}\right)_{i \in I}$ is initial with respect to $|-|:$ Top $\rightarrow$ Set.

Proof. The equivalence (i) $\Leftrightarrow($ iii) follows from Theorem 2.5 and Proposition 2.3 , and (i) $\Leftrightarrow(i i)$ follows from the fact that every monosource in CompHaus is initial with respect to the canonical forgetful functor $|-|:$ CompHaus $\rightarrow$ Set.

In the sequel we call a mono-source in StablyComp initial if it is initial with respect to any of the above-mentioned forgetful functors, and similarly for mono-sources in OrdCompHaus sep.

A stably compact space $X$ is called spectral whenever the source $(X \rightarrow 2)$ of all spectral maps from $X$ into the Sierpiński space $2=\{0,1\}$ (with $\{1\}$ open) is point-separating and initial, that is, the compact open subsets of $X$ form a basis for the topology. We let Spec denote the category of spectral spaces and spectral maps. For each object $X$ in StablyComp, the canonical (surjection,initial mono-source)-factorisation of the source $(X \rightarrow 2)$ in StablyComp provides a Spec-reflection of $X$ (see [Adámek et al., 1990, Theorem 16.8]), therefore Spec is a reflective subcategory of StablyComp closed under initial mono-sources.

Correspondingly, an anti-symmetric ordered compact Hausdorff space $X$ is a Priestley space (see [Priestley, 1970, 1972]) whenever the source $(X \rightarrow 2)$ of all continuous monotone maps from $X$ into $2=\{0 \leq 1\}$ (with the discrete topology) is point-separating and initial. Hence, Spec is

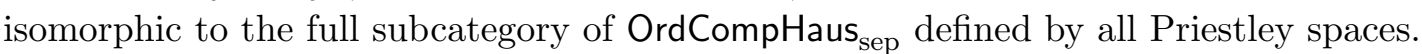

A compact Hausdorff space $X$ is a Stone space whenever the source $(X \rightarrow 2)$ of all continuous maps from $X$ into the discrete space $2=\{0,1\}$ is point-separating (and hence initial with respect to $|-|$ : CompHaus $\rightarrow$ Set), which amounts to saying that the simultaneously open and closed subsets of $X$ form a basis for the topology. Hence, a topological space $X$ is a Stone space if and only if $X$ is spectral and Hausdorff. As above, Adámek et al., 1990, Theorem 16.8] guarantees that the inclusion functor Stone $\rightarrow$ CompHaus has a left adjoint. By definition, the functor $|-|:$ StablyComp $\rightarrow$ CompHaus restricts to $|-|:$ Spec $\rightarrow$ Stone which has the inclusion functor Stone $\hookrightarrow$ Spec as a left adjoint. We note that an ordered compact Hausdorff space $X$ with underlying Stone space $|X|$ need not be a Priestley space (see [Stralka, 1980]). 
The lower Vietoris monad $\mathbb{V}=(V, e, m)$ on Top consists of the functor $V:$ Top $\rightarrow$ Top which sends a topological space $X$ to the space

$$
V X=\{A \subseteq X \mid A \text { is closed }\}
$$

with the topology generated by the sets

$$
U^{\diamond}=\{A \in V X \mid A \cap U \neq \varnothing\} \quad(U \subseteq X \text { open }),
$$

and $V f: V X \rightarrow V Y$ sends $A$ to $\overline{f[A]}$, for $f: X \rightarrow Y$ in Top; and the unit $e$ and the multiplication $m$ of $\mathbb{V}$ are given by

$$
e_{X}: X \rightarrow V X, x \mapsto \overline{\{x\}} \quad \text { and } \quad m_{X}: V V X \rightarrow V X, \mathcal{A} \mapsto \bigcup \mathcal{A}
$$

respectively. Since $\bigcup_{i \in I} U_{i}^{\diamond}=\left(\bigcup_{i \in I} U_{i}\right)^{\diamond}$ it is enough to consider basic opens of $X$ in the definition of the topology of $V X$. The underlying order of $V X$ is the opposite of subset inclusion, that is, $A \leq B$ if and only if $A \supseteq B$, for all $A, B \in V X$. Also note that, for all $U \subseteq X$ open and $\mathcal{A} \subseteq V X$ closed,

$$
e_{X}^{-1}\left(U^{\diamond}\right)=U, \quad \quad \bigcup \mathcal{A}=e_{X}^{-1}(\mathcal{A}), \quad m_{X}^{-1}\left(U^{\diamond}\right)=\left(U^{\diamond}\right)^{\diamond} .
$$

Lemma 2.7. For topological spaces $X_{1}$ and $X_{2}, V\left(X_{1}+X_{2}\right) \simeq V X_{1} \times V X_{2}$.

Proof. We put $f_{1}: V\left(X_{1}+X_{2}\right) \rightarrow V X_{1}, C \mapsto C \cap X_{1}$ and $f_{2}: V\left(X_{1}+X_{2}\right) \rightarrow V X_{2}, C \mapsto C \cap X_{2}$. For open subsets $U_{1} \subseteq X_{1}$ and $U_{2} \subseteq X_{2}$,

$$
f_{1}^{-1}\left(U_{1}^{\diamond}\right)=\left\{C \in V\left(X_{1}+X_{2}\right) \mid C \cap U_{1} \neq \varnothing\right\}
$$

and

$$
f_{2}^{-1}\left(U_{2}^{\diamond}\right)=\left\{C \in V\left(X_{1}+X_{2}\right) \mid C \cap U_{2} \neq \varnothing\right\},
$$

hence both $f_{1}$ and $f_{2}$ are continuous. The induced continuous map $f: V\left(X_{1}+X_{2}\right) \rightarrow V X_{1} \times V X_{2}$ is bijective with inverse $g: V X_{1} \times V X_{2} \rightarrow V\left(X_{1}+X_{2}\right),\left(A_{1}, A_{2}\right) \mapsto A_{1}+A_{2}$. For an open subset $W \subseteq X_{1}+X_{2}$ we put $U_{1}=W \cap X_{1}$ and $U_{2}=W \cap X_{2}$, and observe that

$$
g^{-1}(W)=\left(U_{1}^{\diamond} \times V X_{2}\right) \cup\left(V X_{1} \times U_{2}^{\diamond}\right)
$$

is open in $V X_{1} \times V X_{2}$. Consequently, $g$ is continuous as well.

Lemma 2.8. For topological spaces $X_{1}$ and $X_{2}$, the map

$$
\Pi: V\left(X_{1}\right) \times V\left(X_{2}\right) \rightarrow V\left(X_{1} \times X_{2}\right),\left(A_{1}, A_{2}\right) \mapsto A_{1} \times A_{2}
$$

is continuous and left adjoint to $\pi:=\left\langle V \pi_{1}, V \pi_{2}\right\rangle: V\left(X_{1} \times X_{2}\right) \rightarrow V\left(X_{1}\right) \times V\left(X_{2}\right)$. Hence, if $V\left(X_{1}\right)$ and $V\left(X_{2}\right)$ are stably compact spaces, then $\Pi$ is spectral.

Proof. For open subsets $U_{1} \subseteq X_{1}$ and $U_{2} \subseteq X_{2}$,

$$
\Pi^{-1}\left(\left(U_{1} \times U_{2}\right)^{\diamond}\right)=U_{1}^{\diamond} \times U_{2}^{\diamond},
$$

hence $\Pi$ is continuous. Given now $\left(A_{1}, A_{2}\right) \in V\left(X_{1}\right) \times V\left(X_{2}\right)$ and $W \in V\left(X_{1} \times X_{2}\right)$, we find

$$
(A, B) \leq \pi \cdot \Pi(A, B) \quad \text { and } \quad W \geq \Pi \cdot \pi(W),
$$

hence $\Pi \dashv \pi$.

Below we record some important properties of the lower Vietoris space $V X$. For more information we refer to Schalk 1993.

Proposition 2.9. (1) For every topological space $X, V X$ is sober.

(2) A topological space $X$ is core-compact if and only if $V X$ is stably compact. Hence, if $X$ is sober, then $X$ is locally compact if and only if $V X$ is stably compact.

(3) If $f: X \rightarrow Y$ is a spectral map between stably compact spaces, then $V f: V X \rightarrow V Y$ is spectral

(4) If $X$ is stably compact, then $e_{X}: X \rightarrow V X$ and $m_{X}: V V X \rightarrow V X$ are spectral. 
(5) A stably compact space $X$ is spectral if and only if $V X$ is spectral.

In particular, the monad $\mathbb{V}=(V, e, m)$ on Top restricts to a monad on Spec, also denoted as $\mathbb{V}=(V, e, m)$. We cannot restrict $\mathbb{V}$ further to Stone since $V X$ is not even $\mathrm{T}_{1}$, except for $X=\varnothing$. However, we can transfer $\mathbb{V}$ to the Vietoris monad $\hat{\mathbb{V}}=(\hat{V}, e, m)$ on Stone via the adjunction Spec $\rightleftarrows$ Stone. Explicitly, $\hat{V} X$ is again the set of all closed subsets of $X$ but now with the topology generated by the sets

$$
U^{\diamond} \quad(U \subseteq X \text { open }) \quad \text { and } \quad\{A \subseteq X \text { closed } \mid A \cap K=\varnothing\} \quad(K \subseteq X \text { compact }) ;
$$

this is indeed the topology originally considered by [Vietoris, 1922]. The unit $e$ and the multiplication $m$ are defined as above, but note that $e_{X}(x)=\{x\}$ since $X$ is Hausdorff.

\section{Kleisli dualities}

The aim of this section is to explain how Theorem 1.12 can be used to extend duality results to larger categories. Motivated by our main examples, we consider here natural dualities (see Dimov and Tholen [1989], (Porst and Tholen, 1991] and [Clark and Davey, 1998]) with a category of algebras on one side.

Assumption A. Throughout this section we let $\mathrm{X}$ be a category equipped with a faithful functor $|-|: X \rightarrow$ Set. Moreover, we fix an object $\tilde{X}$ in $\mathbf{X}$ and assume that $\mathbf{X}$ has all powers of $\tilde{X}$ and all equalisers of pairs of morphisms between powers of $\tilde{X}$, and that $|-|$ preserves these limits. Furthermore, let $\Omega=\left(\Omega_{0}, \delta\right)$ be a signature, that is, $\Omega_{0}$ is a class (of operation symbols) and $\delta: \Omega_{0} \rightarrow$ Card assigns to each operation symbol its arity. We write $\operatorname{Alg}(\Omega)$ to denote the category of $\Omega$-algebras and $\Omega$-homomorphisms. We also assume that an $\Omega$-algebra $\tilde{B}$ is given with $|\tilde{X}|=|\tilde{B}|$ and so that, for every operation symbol from $\Omega$ with arity $k$, the operation $|\tilde{B}|^{k} \rightarrow|\tilde{B}|$ underlies an X-morphism $\tilde{X}^{k} \rightarrow \tilde{X}$.

The following result is well-known in duality theory; see [Johnstone, 1986, VI.4], for instance.

Theorem 3.1. Under the assumption $(\mathrm{A})$, there is an adjunction

$$
(F \dashv G, \eta, \varepsilon): \operatorname{Alg}(\Omega)^{\mathrm{op}} \rightleftarrows \mathrm{X}
$$

so that

$$
|G|=\operatorname{Alg}(\Omega)(-, \tilde{B}) \quad \text { and } \quad|F|=\mathrm{X}(-, \tilde{X})
$$

and, for every object $B$ in $\operatorname{Alg}(\Omega)$ and every object $X$ in $\mathrm{X}$, the objects $G B$ and $F X$ have the | - |-initial structure with respect to the sources

$$
\left(\operatorname{Alg}(\Omega)(B, \tilde{B}) \stackrel{\mathrm{ev}_{x}}{\longrightarrow}|\tilde{B}|=|\tilde{X}|, h \mapsto h(x)\right)_{x \in|B|}
$$

respectively

$$
\left(\mathrm{X}(X, \tilde{X}) \stackrel{\mathrm{ev}_{x}}{\longrightarrow}|\tilde{X}|=|\tilde{B}|, h \mapsto h(x)\right)_{x \in|X|} .
$$

Moreover, both $\eta_{X}$ and $\varepsilon_{B}$ send $x$ to the point evaluation map ev defined above.

We denote by $\operatorname{Fix}(\Omega)$ the full subcategory of $\operatorname{Alg}(\Omega)$ defined by those $\Omega$-algebras $B$ where $\varepsilon_{B}$ is an isomorphism.

Let now $\Omega^{\prime}=\left(\Omega_{0}^{\prime}, \delta^{\prime}\right)$ be a sub-signature of $\Omega$; that is, $\Omega_{0}^{\prime} \subseteq \Omega_{0}$ and $\delta^{\prime}$ is the restriction of $\delta$ to $\Omega_{0}^{\prime}$. Note that there is a canonical forgetful functor $\operatorname{Alg}(\Omega) \rightarrow \operatorname{Alg}\left(\Omega^{\prime}\right)$. Applying the result above to $\Omega^{\prime}$ in lieu of $\Omega$ yields an adjunction

$$
\left(F^{\prime} \dashv G^{\prime}, \eta^{\prime}, \varepsilon^{\prime}\right): \operatorname{Alg}\left(\Omega^{\prime}\right)^{\mathrm{op}} \rightleftarrows \mathrm{X} .
$$

We denote by $\operatorname{Fix}(\Omega)_{\Omega^{\prime}}$ the full subcategory of $\operatorname{Alg}\left(\Omega^{\prime}\right)$ defined by those $\Omega^{\prime}$-algebras which underlie an $\Omega$-algebra in $\operatorname{Fix}(\Omega)$. 
Lemma 3.2. Assume that $F X$ is in $\operatorname{Fix}(\Omega)$, for every object $X$ in $\mathrm{X}$. Then the adjuntion (2) restricts to a Kleisli adjunction

$$
\left(F^{\prime} \dashv G^{\prime}, \eta^{\prime}, \varepsilon^{\prime}\right): \operatorname{Fix}(\Omega)_{\Omega^{\prime}}^{\mathrm{op}} \rightleftarrows \mathrm{X} .
$$

Note that the assumption in the lemma above can be always obtained by replacing $X$ with its full subcategory of those objects $X$ where $\eta_{X}$ is an isomorphism. In order to identify the induced monad with a more familiar one, we consider now the following situation.

Assumption B. Assume that the functor $F: \mathrm{X} \rightarrow \operatorname{Alg}(\Omega)$ of Theorem 3.1 takes value in $\operatorname{Fix}(\Omega)$. Let $\Omega^{\prime}=\left(\Omega_{0}^{\prime}, \delta^{\prime}\right)$ be a sub-signature of $\Omega$ and let $\mathbb{T}=(T, e, m)$ be a monad on $\mathrm{X}$ with $T\left(X_{1}\right) \simeq \tilde{X}$, for some object $X_{1}$ in $\mathrm{X}$. To simplify notation, we assume that $\tilde{X}$ is chosen so that $T\left(X_{1}\right)=\tilde{X}$. Hence, $\tilde{X}$ is a $\mathbb{T}$-algebra, and therefore every $h: X \rightarrow \tilde{X}$ has a unique extension $\bar{h}: T X \rightarrow \tilde{X}$ where $\bar{h}$ is a $\mathbb{T}$-homomorphism with $\bar{h} \cdot e_{X}=h$. For every object $X$ in $\mathbf{X}$ we assume:

- the map

$$
(\stackrel{-}{-}): \mathrm{X}(X, \tilde{X}) \rightarrow \mathrm{X}(T X, \tilde{X}), h \mapsto \bar{h}
$$

preserves all operations from $\Omega^{\prime}$, that is, $\left(_{-}^{-}\right)$is a morphism in $\operatorname{Fix}(\Omega)_{\Omega^{\prime}}$ of type $F^{\prime} X \rightarrow$ $F^{\prime} T X$, and

- the source $(\bar{h}: T X \rightarrow \tilde{X})_{h \in \mathrm{X}(X, \tilde{X})}$ is point-separating and $\mid$ - |-initial.

Proposition 3.3. Under the assumptions $\mathrm{A}$ e $(\mathrm{B})$, the hom-functor $\mathrm{X}_{\mathbb{T}}\left(-, X_{1}\right)$ lifts to a functor $J: \mathrm{X}_{\mathbb{T}} \rightarrow \mathrm{B}_{\Omega^{\prime}}^{\text {op }}$ so that the diagram

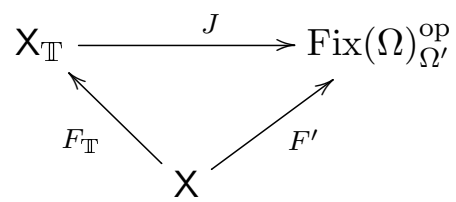

commutes. The induced monad morphism $j$ is component-wise an embedding with $X$-component

$$
j_{X}: T X \rightarrow G^{\prime} F^{\prime} X, \mathfrak{x} \mapsto(h \mapsto \bar{h}(\mathfrak{x})),
$$

for each object $X$ in $\mathbf{X}$.

Proof. For each object $X$ in $\mathrm{X}_{\mathbb{T}}$, one has $\mathrm{X}_{\mathbb{T}}\left(X, X_{1}\right)=\mathrm{X}(X, \tilde{X})$, and we put $J X=F^{\prime} X$. Given a morphism $r: X \longrightarrow Y$ in $\mathrm{X}_{\mathbb{T}}$, the map $\mathrm{X}_{\mathbb{T}}\left(r, X_{1}\right): \mathrm{X}_{\mathbb{T}}\left(Y, X_{1}\right) \rightarrow \mathrm{X}_{\mathbb{T}}\left(X, X_{1}\right)$ sends $h \in \mathrm{X}_{\mathbb{T}}\left(Y, X_{1}\right)=\mathrm{X}(Y, \tilde{X})$ to $m_{X_{1}} \cdot T h \cdot r$. Hence, this map can be written as the composite

$$
\mathrm{X}(Y, \tilde{X}) \stackrel{(-)}{\longrightarrow} \mathrm{X}(T Y, \tilde{X}) \stackrel{\mathrm{X}(r, \tilde{X})}{\longrightarrow} \mathrm{X}(X, \tilde{X})
$$

and therefore underlies a morphism $J r: J Y \rightarrow J X$ in $\operatorname{Fix}(\Omega)_{\Omega^{\prime}}$. In conclusion, this construction yields a functor $J: X_{\mathbb{T}} \rightarrow \operatorname{Fix}(\Omega)_{\Omega^{\prime}}^{\text {op }}$ which clearly satisfies $J F_{\mathbb{T}}=F^{\prime}$. To describe the corresponding natural transformation $\iota: G_{\mathbb{T}} \rightarrow G^{\prime} J$, we note that $\eta_{G_{\mathbb{T}} X}^{\prime}$ is given by the map

$$
\eta_{G_{\mathbb{T}} X}^{\prime}: T X \rightarrow \operatorname{Fix}(\Omega)_{\Omega^{\prime}}(\mathrm{X}(T X, \tilde{X}), \tilde{B}), \mathfrak{x} \mapsto \mathrm{ev}_{\mathfrak{x}},
$$

$\varepsilon_{X}: T X \longrightarrow X$ of $F_{\mathbb{T}} \dashv G_{\mathbb{T}}$ corresponds to the X-morphism $1_{T} X: T X \rightarrow T X$ and therefore $J \varepsilon_{X}$ sends $h \in \mathrm{X}(X, \tilde{X})$ to $\bar{h}$, and $G^{\prime} J \varepsilon_{X}$ sends $\Phi: J T X \rightarrow \tilde{B}$ to the $\operatorname{map} \mathrm{X}(X, \tilde{X}) \rightarrow \tilde{B}, h \mapsto \Phi(\bar{h})$. Putting all together, $\iota_{X}: G_{\mathbb{T}} X \rightarrow G^{\prime} J X$ sends $\mathfrak{x} \in T X$ to the map $\mathrm{X}(X, \tilde{X}) \rightarrow \tilde{B}, h \mapsto \bar{h}(\mathfrak{x})$; and from $j_{X}=\iota_{F_{\mathbb{T}} X}$ we obtain the desired description of $j$. Finally, for every object $X$ in $\mathrm{X}$ and every morphism $h: X \rightarrow \tilde{X}$ in $\mathrm{X}$, the diagram

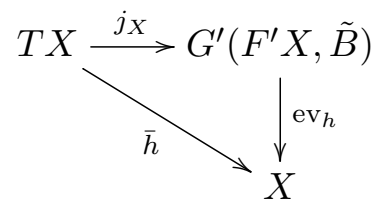

commutes and therefore $j_{X}: T X \rightarrow G^{\prime} F^{\prime} X$ is an embedding in $\mathrm{X}$. 
By construction, the diagram

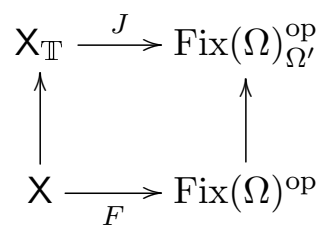

of functors commutes. If both $J$ and $F$ are equivalence functors, then a category constructed from $X \rightarrow X_{\mathbb{T}}$ is dually equivalent to the category obtained from $\operatorname{Fix}(\Omega) \rightarrow \operatorname{Fix}(\Omega)_{\Omega^{\prime}}$ by the same construction. For instance, the category $\operatorname{Coalg}(T: X \rightarrow \mathbf{X})$ of coalgebras for $T$ has as objects X-morphisms $e: X \rightarrow T X$, and a morphism $f:(X, e) \rightarrow\left(X^{\prime}, e^{\prime}\right)$ in $\operatorname{Coalg}(T: \mathbf{X} \rightarrow \mathbf{X})$ is a X-morphism $f: X \rightarrow X^{\prime}$ with $T f \cdot e=e^{\prime} \cdot f$. Equivalently, we can think of the objects of Coalg $(T: \mathrm{X} \rightarrow \mathrm{X})$ as endomorphisms $e: X \longrightarrow X$ in $\mathrm{X}_{\mathbb{T}}$, and $T f \cdot e=e^{\prime} \cdot f$ means precisely that the diagram

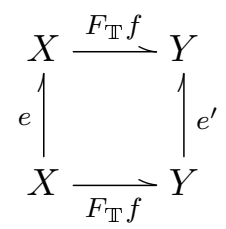

commutes in $X_{\mathbb{T}}$. Therefore:

Corollary 3.4. Assume that $J: \mathrm{X}_{\mathbb{T}} \rightarrow \operatorname{Fix}(\Omega)_{\Omega^{\prime}}^{\mathrm{op}}$ and $F: \mathrm{X} \rightarrow \operatorname{Fix}(\Omega)^{\mathrm{op}}$ are equivalence functors. Then the category $\operatorname{Coalg}(T: \mathrm{X} \rightarrow \mathrm{X})$ is dually equivalent to the category $\operatorname{Fix}(\Omega)\left[\Omega^{\prime}\right]$ of " $\Omega$-algebras with operator", that is, the objects of $\operatorname{Fix}(\Omega)\left[\Omega^{\prime}\right]$ are $\operatorname{Fix}(\Omega)$-objects $B$ equipped with an unitary operation $h: B \rightarrow B$ which preserves all operations from $\Omega^{\prime}$, and a morphism $f:(B, h) \rightarrow\left(B^{\prime}, h^{\prime}\right)$ in $\operatorname{Fix}(\Omega)\left[\Omega^{\prime}\right]$ is a $\Omega$-homomorphism $f: B \rightarrow B^{\prime}$ with $h^{\prime} \cdot f=f \cdot h$.

We apply now the techniques explained above to well-known dualities to obtain duality results for categories of "algebras with an operator". Most of these dualities are well-known; however, our objective here is to show how to obtain them in a uniform manner.

Examples 3.5. (1) We consider first $X=$ Set with $\tilde{X}=2=\{0,1\}$, and the category CABool of complete atomic Boolean algebras and functions preserving all suprema and all infima with $\tilde{B}=$ 2 being the two-element Boolean algebra. It is well-known that the corresponding adjunction

$$
(F \dashv G, \eta, \varepsilon): \text { CABool }^{\text {op }} \rightleftarrows \text { Set }
$$

is actually an equivalence (see [Johnstone, 1986], for instance). We consider now the category CABool $\vee$ of complete atomic Boolean algebras and functions preserving all suprema as well as the powerset monad $\mathbb{P}=(P, e, m)$ on Set. Clearly, $P 1=2$, and, for every map $h: X \rightarrow 2$, the extension $\bar{h}: P X \rightarrow 2$ is defined by

$$
\bar{h}(A)=1 \Longleftrightarrow \exists x \in A \cdot h(x)=1,
$$

for all $A \subseteq X$. In other words, $\bar{h}(A)=\bigvee\{h(x) \mid x \in A\}$, and we conclude that the map $\left({ }^{-}\right)$ of Assumption $B$ preserves suprema. A quick calculation shows that the monad morphism $j$ induced by $J$ is given by

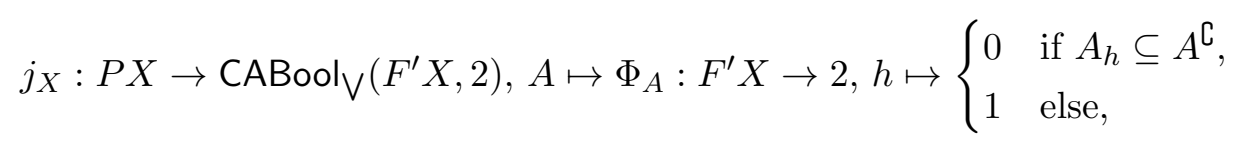

where $A_{h}=h^{-1}(1)$. Since every suprema-preserving map $\Phi: F^{\prime} X \rightarrow 2$ is completely determined by the largest element $h \in F^{\prime} X$ with $\Phi(h)=0$, each component of $j$ is surjective. Therefore, by Proposition 3.3, $j$ is an isomorphism. Finally, from Theorem 1.12 and Corollary 3.4 we obtain:

$$
\text { Rel } \simeq \text { CABool } V_{V}^{\text {op }} \quad \text { and } \quad \operatorname{Coalg}(P: \text { Set } \rightarrow \text { Set }) \simeq \text { CABool }[\bigvee]^{\text {op }} \text {. }
$$


Here CABool [V] denotes the category with objects complete atomic Boolean algebras $B$ equipped with a unary operation $h: B \rightarrow B$ which preserves suprema, and a morphism $h:(B, h) \rightarrow$ $\left(B^{\prime}, h^{\prime}\right)$ in CABool [V] is a morphism $h: B \rightarrow B^{\prime}$ in CABool with $f \cdot h=h^{\prime} \cdot f$.

(2) Similarly, if we consider the category $\mathrm{CABool}_{\top, \wedge}$ of complete atomic Boolean algebras and finite infima preserving maps instead, the adjunction

$$
\left(F^{\prime} \dashv G^{\prime}, \eta^{\prime}, \varepsilon^{\prime}\right): \text { CABool }{ }_{\top, \wedge}^{\text {op }} \rightleftarrows \text { Set }
$$

induces a monad isomorphic to the filter monad $\mathbb{F}$ on Set. Hence:

$$
\operatorname{Set}_{\mathbb{F}} \simeq \text { CABool }_{T, \wedge}^{\text {op }} \quad \text { and } \quad \operatorname{Coalg}(F: \text { Set } \rightarrow \text { Set }) \simeq \operatorname{CABool}[T, \wedge]^{\text {op }} .
$$

(3) Our next example involves $X=$ Top and the category Frm of frames and frame-homomorphisms. Moreover, $\tilde{X}=2=\{0,1\}$ is the Sierpiński space with $\{1\}$ open and $\tilde{B}=2$ is the two-element frame. We obtain the adjunction

$$
(F \dashv G, \eta, \varepsilon): \mathrm{Frm}^{\mathrm{op}} \rightleftarrows \mathrm{Top}
$$

where $\varepsilon_{B}$ is an isomorphism if and only if the frame $B$ is spatial, and $\eta_{X}$ is an isomorphism if and only if the topological space $X$ is sober. Similar to the previous example, we consider the category $\mathrm{SFrm}_{\mathrm{T}, \wedge}$ of spatial frames and finite infima preserving maps, and obtain a Kleisli adjunction

$$
\left(F^{\prime} \dashv G^{\prime}, \eta^{\prime}, \varepsilon^{\prime}\right): \mathrm{SFrm}_{\mathrm{T}, \wedge}^{\mathrm{op}} \rightleftarrows \text { Top. }
$$

It is well-known that the induced monad is isomorphic to the filter monad $\mathbb{F}=\left(F_{\tau}, e, m\right)$ on Top. In fact, $F_{\tau} 1$ is the Sierpiński space, and

$$
\left({ }^{-}\right): \operatorname{Top}(X, 2) \rightarrow \operatorname{Top}\left(F_{\tau} X, 2\right)
$$

sends $h: X \rightarrow 2$ the characteristic map of $\left(U_{h}\right)^{\#}=\left\{\mathfrak{f} \in F_{\tau} X \mid U_{h} \in \mathfrak{f}\right\}$ (where $U_{h}=h^{-1}(1)$ ), hence $\left({ }^{-}\right)$preserves finite infima. By definition, the topology on $F_{\tau} X$ is generated by all sets $\left(U_{h}\right)^{\#}$ for $h: X \rightarrow 2$ continuous, which tells us that the point-separating source $\left(\bar{h}: F_{\tau} X \rightarrow\right.$ $2)_{h \in \operatorname{Top}(X, 2)}$ is initial. The $X$-component of the monad morphism $j$ is given by

$$
j_{X}: F_{\tau} X \rightarrow \operatorname{SFrm}_{\top, \wedge}\left(F^{\prime} X, 2\right), \mathfrak{f} \mapsto \Phi_{\mathfrak{f}}: F^{\prime} X \rightarrow 2, h \mapsto \begin{cases}1 & U_{h} \in \mathfrak{f}, \\ 0 & \text { else },\end{cases}
$$

hence $j_{X}$ is surjective and therefore an isomorphism. In conclusion:

$$
\operatorname{Top}_{\mathbb{F}} \simeq \mathrm{SFrm}_{\top, \wedge} \simeq \operatorname{Sob}_{\mathbb{F}} \quad \text { and } \quad \operatorname{Coalg}(F: \mathrm{Sob} \rightarrow \mathrm{Sob}) \simeq \operatorname{SFrm}[\top, \wedge]^{\text {op }} .
$$

Note that the second equivalence is only valid for $F$ restricted to the category Sob of sober spaces and continuous maps since Corollary 3.4 assumes that the adjunction of Theorem 3.1 is an equivalence.

(4) We substitute now in the example above $\mathrm{SFrm}_{\mathrm{T}, \wedge}$ with the category $\mathrm{SFrm}_{\bigvee}$ of spatial frames and suprema preserving maps. We show that the monad induced by the adjunction

$$
\left(F^{\prime} \dashv G^{\prime}, \eta^{\prime}, \varepsilon^{\prime}\right): \text { SFrm } \mathrm{Vp} \rightleftarrows \text { Top. }
$$

is isomorphic to the lower Vietoris monad $\mathbb{V}=(V, e, m)$ on Top (see Section 2). Clearly, $V 1$ is the Sierpiński space, and the map

$$
(\stackrel{-}{-}): \operatorname{Top}(X, 2) \rightarrow \operatorname{Top}(V X, 2)
$$

sends $h: X \rightarrow 2$ to the characteristic map of $\left(U_{h}\right)^{\diamond}$. Therefore $\left(^{-}\right)$preserves all suprema and $V X$ has by definition the initial topology with respect to the point-separating source $(\bar{h}: V X \rightarrow$ $2)_{h \in \operatorname{Top}(X, 2)}$. Similar to the first example, the monad morphism $j$ induced by $J$ is given by

$$
j_{X}: V X \rightarrow \operatorname{SFrm}_{\bigvee}\left(F^{\prime} X, 2\right), A \mapsto \Phi_{A}: F^{\prime} X \rightarrow 2, h \mapsto \begin{cases}0 & \text { if } U_{h} \subseteq A^{\complement}, \\ 1 & \text { else, }\end{cases}
$$


hence $j$ is an isomorphism and we obtain:

$$
\mathrm{Top}_{\mathbb{V}} \simeq \mathrm{SFrm}_{\mathrm{V}}^{\mathrm{op}} \simeq \mathrm{Sob}_{\mathbb{V}} \quad \text { and } \quad \operatorname{Coalg}(V: \mathrm{Sob} \rightarrow \mathrm{Sob}) \simeq \mathrm{SFrm}[\bigvee]^{\text {op }} .
$$

Clearly, a morphism $X \longrightarrow Y$ in Top $_{\mathbb{V}}$ corresponds to a relation $X \mapsto Y$, and we call a relation $r: X \mapsto Y$ between topological spaces continuous whenever the corresponding map $\ulcorner r\urcorner: X \rightarrow P Y$ factors as $X \stackrel{\ulcorner r\urcorner}{\longrightarrow} V Y \hookrightarrow P Y$ and, moreover, $\left.{ }^{\ulcorner} r\right\urcorner: X \rightarrow V Y$ is continuous. For

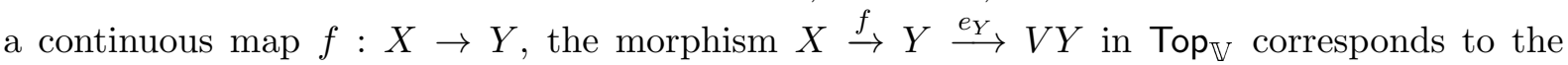
continuous relation

$$
f_{*}: X \mapsto Y, x f_{*} y \Longleftrightarrow f(x) \leq y,
$$

where $\leq$ refers to the underlying order of $Y$. We also note that every continuous relation $r: X \mapsto Y$ satisfies

$$
\left(x \leq x^{\prime} r y^{\prime} \leq y\right) \Rightarrow x r y
$$

for all $x, x^{\prime} \in X$ and $y, y^{\prime} \in Y$.

(5) We consider now the category $X=$ Spec of spectral spaces and spectral maps. As shown in Stone, 1938, Spec is equivalent to the dual of the category DLat of distributive lattices (with top and bottom element) and lattice homomorphisms, and this equivalence is induced by $\tilde{X}=2$ the Sierpiński space and $\tilde{B}=2$ the two-element lattice. With DLat ${ }_{\perp, \vee}$ denoting the category of distributive lattices and finite suprema preserving maps, we obtain a Kleisli adjunction

$$
\left(F^{\prime} \dashv G^{\prime}, \eta^{\prime}, \varepsilon^{\prime}\right): \mathrm{DLat}_{\perp, \mathrm{V}}^{\mathrm{op}} \rightleftarrows \text { Spec. }
$$

For the lower Vietoris monad $\mathbb{V}=(V, e, m)$ on Spec, $V 1$ is the Sierpinski space and the map $\left(_{-}^{-}\right): \operatorname{Spec}(X, 2) \rightarrow \operatorname{Spec}(V X, 2)$ is the restriction of the corresponding map of Example 4 above and therefore preserves finite suprema; $(\bar{h}: V X \rightarrow 2)_{h \in \operatorname{Spec}(X, 2)}$ is point-separating and $V X$ has the initial topology (= initial Spec-structure). As above, the monad morphism $j$ induced by $J$ is given by

$$
j_{X}: V X \rightarrow \operatorname{DLat}_{\perp, \mathrm{V}}\left(F^{\prime} X, 2\right), A \mapsto \Phi_{A}: F^{\prime} X \rightarrow 2, h \mapsto \begin{cases}0 & \text { if } U_{h} \subseteq A^{\complement}, \\ 1 & \text { else, }\end{cases}
$$

and compactness of the sets $U_{h}$ (for $h: X \rightarrow 2$ in Spec) assures that $j_{X}$ is surjective. Therefore:

$$
\operatorname{Spec}_{\mathbb{V}} \simeq \text { DLat }_{\perp, V}^{\text {op }} \quad \text { and } \quad \operatorname{Coalg}(V: \operatorname{Spec} \rightarrow \text { Spec }) \simeq \text { DLat }[\perp, V]^{\text {op }} .
$$

The above mentioned dualities were also obtained in [Cignoli et al., 1991], [Petrovich, 1996] and Bonsangue et al. 2007]. Put differently, SpecRel $\simeq \mathrm{DLat}_{\perp, \vee}^{\mathrm{op}}$ where SpecRel denotes the category of spectral spaces and spectral relations (that is, relations $r: X \mapsto Y$ between spectral spaces so that $\ulcorner r\urcorner: X \rightarrow P Y$ factors as $X \stackrel{\ulcorner r\urcorner}{\longrightarrow} V Y \hookrightarrow P Y$ and $\ulcorner r\urcorner: X \rightarrow V Y$ is spectral) with relational composition.

(6) Finally, we describe the dualities involving Boolean algebras mentioned in the Introduction. By Stone, 1936, $\mathrm{X}=$ Stone is equivalent to the dual of the category Bool of Boolean algebras and homomorphisms. As above, for the monad $\hat{\mathbb{V}}=(\hat{V}, e, m)$ on Stone one obtains

$$
\text { StoneRel } \simeq \text { Stone }_{\hat{\mathbb{V}}} \simeq \text { Bool }_{\perp, \vee}^{\text {op }} \quad \text { and } \quad \text { Coalg }(\hat{V}: \text { Stone } \rightarrow \text { Stone }) \simeq \text { Bool }[\perp, \vee]^{\text {op }} .
$$

Here StoneRel denotes full subcategory of SpecRel defined by all Stone spaces.

\section{Monoidal Structures}

The starting point of this section is the observation that the topological product of two spectral spaces is not their product in SpecRel, as it can be seen already in the simplest case:

$$
\operatorname{SpecRel}(1,1 \times 1) \not \varkappa \operatorname{SpecRel}(1,1) \times \operatorname{SpecRel}(1,1) .
$$

In this section we shall see that this operation corresponds on the algebraic side to a tensor product which represents bimorphisms. 
The formula above suggests that the product $1 \times 1$ in SpecRel should be the two-element discrete space. In fact, similar to the situation for Rel and Set, products in SpecRel are given by coproducts in Spec. We will need a piece of notation. For a morphism $f: X \rightarrow Y$ in Spec, we define a relation $f^{*}: Y \longrightarrow X$ as $y f^{*} x$ whenever $y \leq f(x)$, for all $x \in X$ and $y \in Y$. We emphasize that $f^{*}$ does not need to be a spectral relation; however, if $f$ is an open map, then $f^{*}$ is spectral (see [Hofmann, 2014, Proposition 8.4]). Also note that, for a morphism $r: Z \longrightarrow Y$ in SpecRel and $z \in Z, x \in X$,

$$
z\left(f^{*} \cdot r\right) x \Longleftrightarrow \exists y \in Y .(z r y) \wedge(y \leq f(x)) \Longleftrightarrow z r f(x) .
$$

Lemma 4.1. For spectral spaces $X_{1}$ and $X_{2}$, the product of $X_{1}$ and $X_{2}$ in SpecRel is given by their topological sum (which is also their coproduct in Spec and in SpecRel). If $i_{1}: X_{1} \rightarrow$ $X_{1}+X_{2}$ and $i_{2}: X_{2} \rightarrow X_{1}+X_{2}$ denote the coproduct injections, then $i_{1}^{*}: X_{1}+X_{2} \rightarrow X_{1}$ and $i_{2}^{*}: X_{1}+X_{2} \longrightarrow X_{2}$ are the two product projections.

Proof. Given morphisms $r: Z \longrightarrow X_{1}$ and $s: Z \longrightarrow X_{2}$ in SpecRel, let

$$
\langle r, s\rangle: Z \rightarrow X_{1}+X_{2}
$$

be the spectral relation which corresponds to the composite

$$
Z \stackrel{\langle\ulcorner\urcorner\urcorner,\ulcorner s\urcorner\rangle}{\longrightarrow} V\left(X_{1}\right) \times V\left(X_{2}\right) \simeq V\left(X_{1}+X_{2}\right)
$$

in Spec (see Lemma 2.7). Element-wise, for $z \in Z$ and $x \in X_{1}+X_{2}$, one has

$$
z\langle r, s\rangle x \Longleftrightarrow\left(x \in X_{1} \wedge z r x\right) \vee\left(x \in X_{2} \wedge z s x\right) ;
$$

and this gives at once $i_{1}^{*} \cdot\langle r, s\rangle=r$ and $i_{2}^{*} \cdot\langle r, s\rangle=s$. Finally, a spectral relation $t: Z \longrightarrow X_{1}+X_{2}$ with $i_{1}^{*} \cdot t=r$ and $i_{2}^{*} \cdot t=s$ is necessarily described by the right hand side of (3), hence $t=\langle r, s\rangle$.

A similar result holds for StoneRel, and the empty space is an initial and a terminal object in both SpecRel and StoneRel.

Proposition 4.2. The categories SpecRel, StoneRel, DLat ${ }_{\perp, \vee}$ and Bool $_{\perp, \vee}$ have finite products and finite coproducts which coincide.

Note that finite (co)products in the categories $\mathrm{DLat}_{\perp, \vee}$ and $\mathrm{Bool}_{\perp, \vee}$ are given by products in DLat and Bool respectively. For objects $X$ and $Y$ in SpecRel, we denote their topological product as $X \otimes Y$. For spectral relations $r: X \mapsto X^{\prime}$ and $s: Y \rightarrow Y^{\prime}$, we define a relation $r \otimes s: X \otimes Y \rightarrow X^{\prime} \otimes Y^{\prime}$ where $(x, y)(r \otimes s)\left(x^{\prime}, y^{\prime}\right)$ whenever $x r x^{\prime}$ and $r s y^{\prime}$. Then $r \otimes s$ is indeed spectral since, with $\left\ulcorner r^{\urcorner}: X \rightarrow V\left(X^{\prime}\right)\right.$ and \ulcorner\urcorner$\urcorner: Y \rightarrow V\left(Y^{\prime}\right)$ denoting the corresponding spectral maps, $r \otimes s$ corresponds to the map

$$
X \times Y \stackrel{\left.\left\ulcorner^{r}\right\urcorner \times\ulcorner\urcorner\right\urcorner}{\longrightarrow} V\left(X^{\prime}\right) \times V\left(Y^{\prime}\right) \stackrel{\Pi}{\longrightarrow} V\left(X^{\prime} \times Y^{\prime}\right)
$$

in Spec (see Lemma 2.8). One easily verifies that this construction defines a functor

$$
\otimes: \text { SpecRel } \times \text { SpecRel } \rightarrow \text { SpecRel. }
$$

For objects $L, M, N$ in DLat $_{\perp, \vee}$, we call a map $f: L \times M \rightarrow N$ a bimorphism whenever, for all $x \in L, f(x,-): M \rightarrow N$ preserves finite suprema and, for all $y \in M, f(-, y): L \rightarrow N$ preserves finite suprema. Note that a bimorphism is necessarily monotone. We say that an object $P$ in DLat ${ }_{\perp, \vee}$ represents bimorphisms $f: L \times M \rightarrow N$ whenever there is a bimorphism $p: L \times M \rightarrow P$ such that, for every bimorphism $f: L \times M \rightarrow N$, there exists a unique morphism $\bar{f}: P \rightarrow N$ with $\bar{f} \cdot p=f$. If it exists, $P$ is unique up to isomorphism, and we denote such an object as $L \otimes M$ and refer to $p: L \times M \rightarrow L \otimes M$ as the tensor product of $L$ and $M$.

Lemma 4.3. For spectral spaces $X$ and $Y$, the tensor product of $J X$ and $J Y$ exists and is given by

$$
p: J X \times J Y \rightarrow J(X \otimes Y),(A, B) \mapsto A \times B .
$$


Proof. For each spectral space $X$, we identify the elements of $J X$ with the compact open subsets of $X$. Let $X$ and $Y$ be spectral spaces. Clearly, $p: J X \times J Y \rightarrow J(X \otimes Y)$ is a bimorphism. Let $f: S X \times S Y \rightarrow N$ be a bimorphism. It is enough to consider the case $N=S Z$, for a spectral space $Z$. We define a map

$$
g: J(X \otimes Y) \rightarrow P Z, W \mapsto \bigcup\{f(A, B) \mid A \in J X, B \in J Y, A \times B \subseteq W\} .
$$

Then $g$ is monotone, $g(\varnothing)=\varnothing$ and, for all $A \in J X$ and $B \in J Y, g(A \times B)=f(A, B)$. We show now that $g$ preserves binary suprema; since $g$ is monotone, it is enough to show $g\left(W \cup W^{\prime}\right) \subseteq g(W) \cup g\left(W^{\prime}\right)$. To this end, fix $A \in J X$ and $B \in J Y$ with $A \times B \subseteq W \cup W^{\prime}$. Since $W$ and $W^{\prime}$ are compact opens, there are finite sets $I$ and $I^{\prime}$ and compact opens $A_{i}, A_{j}^{\prime} \subseteq X$ and $B_{i}, B_{j}^{\prime} \subseteq Y\left(i \in I, j \in I^{\prime}\right)$ with

$$
W=\bigcup_{i \in I} A_{i} \times B_{i} \quad \text { and } \quad W^{\prime}=\bigcup_{j \in I^{\prime}} A_{j}^{\prime} \times B_{j}^{\prime} .
$$

For $x \in A$, put

$$
A_{x}=A \cap \bigcap\left\{A_{i} \mid i \in I, x \in A_{i}\right\} \cap \bigcap\left\{A_{j}^{\prime} \mid j \in I^{\prime}, x \in A_{j}^{\prime}\right\}
$$

and, for each $y \in B$, put

$$
B_{y}=B \cap \bigcap\left\{B_{i} \mid i \in I, y \in B_{i}\right\} \cap \bigcap\left\{B_{j}^{\prime} \mid j \in I^{\prime}, y \in B_{j}^{\prime}\right\} .
$$

Then $x \in A_{x}, y \in B_{y}$ and $A_{x}$ and $B_{y}$ are open and compact. Hence,

$$
A=\bigcup_{x \in A} A_{x}=A_{x_{1}} \cup \ldots \cup A_{x_{n}} \quad \text { and } \quad B=\bigcup_{y \in B} B_{y}=B_{y_{1}} \cup \ldots \cup B_{y_{m}},
$$

for finitely many elements $x_{1}, \ldots, x_{n} \in A$ and $y_{1}, \ldots, y_{m} \in B$. Since $f$ is a bimorphism, we obtain

$$
f(A, B)=\bigcup\left\{f\left(A_{x_{i}}, B_{y_{j}}\right) \mid 1 \leq i \leq n, 1 \leq j \leq m\right\} .
$$

Let now $z \in f(A, B)$. Then $z \in f\left(A_{x_{k}}, B_{y_{l}}\right)$, for some $1 \leq k \leq n$ and $1 \leq l \leq m$; and $\left(x_{k}, y_{l}\right) \in A \times B \subseteq W \cup W^{\prime}$. Without loss of generality we assume $\left(x_{k}, y_{l}\right) \in W$. We have $x_{k} \in A_{i}$ and $y_{l} \in B_{i}$ for some $i \in I$, hence $A_{x_{k}} \subseteq A_{i}$ and $B_{y_{l}} \subseteq B_{i}$ and therefore

$$
z \in f\left(A_{x_{k}}, B_{y_{l}}\right) \subseteq f\left(A_{i}, B_{i}\right) \subseteq g(W) .
$$

This proves $g\left(W \cup W^{\prime}\right) \subseteq g(W) \cup g\left(W^{\prime}\right)$. From preservation of finite suprema it also follows that $g$ takes values in $J Z$ since (using (4))

$$
g(W)=\bigcup_{i \in I} g\left(A_{i} \times B_{i}\right)=\bigcup_{i \in I} f\left(A_{i}, B_{i}\right)
$$

is compact (and open). This also shows that $g$ is the only finite suprema preserving map $g: J(X \otimes Y) \rightarrow P Z$ with $g \cdot p=f$, and the corestriction $\bar{f}: J(X \otimes Y) \rightarrow J Z$ of $g$ to $J Z$ is the required unique map with $\bar{f} \cdot p=f$.

Theorem 4.4. For all distributive lattices $L$ and $M$, their tensor product in DLat $_{\perp, \vee}$ exists. Moreover, the functor $J:$ SpecRel $\rightarrow$ DLat $_{\perp, \vee}$ satisfies $J(X \otimes Y) \simeq J X \otimes J Y$, for all spectral spaces $X$ and $Y$.

All what was said above can be restricted to Stone spaces and Boolean algebras.

Theorem 4.5. For all Boolean algebras $L$ and $M$, their tensor product in $\mathrm{Boo}_{\perp, \vee} \vee$ exists and the functor $J$ : StoneRel $\rightarrow$ Bool $_{\perp, \vee}$ satisfies $J(X \otimes Y) \simeq J X \otimes J Y$, for all Stone spaces $X$ and $Y$. 
Hence, a spectral relation $X \mapsto X \otimes X$ corresponds to a morphism $B \otimes B \rightarrow B$ in DLat ${ }_{\perp, \mathrm{V}}$, which can be also seen as a map $B \times B \rightarrow B$ preserving finite suprema in each variable (the corresponding result for Boolean algebras can be found in [Celani, 2003]). In fact, the (monoidal) structure on SpecRel and StoneRel can be used to express properties of relations (see Kegelmann, 1999], for instance), and preservation properties of $J$ allow to translate these properties systematically into algebraic properties of the corresponding morphism in DLat ${ }_{\perp, \vee}$ respectively Bool $_{\perp, \vee}$. For instance, the unique map ! : $X \rightarrow 1$ of a Stone space $X$ corresponds to the unique map $2 \rightarrow A$ in Bool sending 0 to $\perp$ and 1 to $\top$, and the diagonal map $\Delta: X \rightarrow X \times X$ corresponds to the map $A \otimes A \rightarrow A$ induced by the bimorphism $\wedge: A \times A \rightarrow A$. A morphism $r: X \mapsto Y$ in StoneRel is a total relation whenever

$$
\forall x \in X \exists y \in Y \text {. } x r y, \quad \text { which is equivalent to }
$$

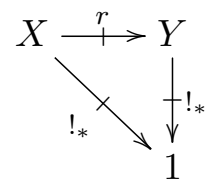

commutes in StoneRel. Hence, $r$ is total if and only if the corresponding morphism $f: B \rightarrow A$ in $\mathrm{Bool}_{\perp, \vee}$ makes the diagram

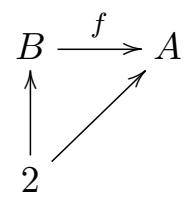

commute, that is, $f$ preserves the top-element. Slightly more general, given two morphisms $r, s: X \mapsto Y$ in StoneRel, we can express the property that each $x \in X$ has a "successor" for at least one of these relations (i.e. $\forall x \in X \exists y \in Y$. $(x r y) \vee(x s y))$ by requiring that

$$
X \stackrel{\langle r, s\rangle}{\longrightarrow} Y+Y \stackrel{!_{*}}{\longmapsto}>1
$$

is equal to $!_{*}: X \rightarrow 1$; and this means precisely that the corresponding morphisms $f, g: B \rightarrow A$ in $\mathrm{Bool}_{\perp, \vee}$ must make the diagram

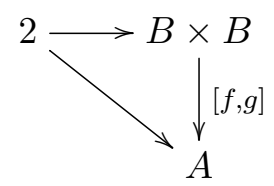

commute, that is, $f(\top) \vee g(\top)=\top$. Similarly, $r: X \mapsto Y$ in StoneRel is a partial map if and only if the diagram

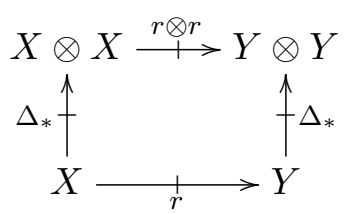

commutes in StoneRel, hence partial maps correspond to binary infima preserving morphisms $f: A \rightarrow B$ in Bool $\perp, \vee$. Finally, a morphism $r: X \rightarrow Y$ in SpecRel is a partial map whenever, for each $x \in X$, the closed set $\{y \in Y \mid x r y\}$ is either empty or has a smallest element with respect to the underlying order of $Y$. The following result can be found in Kegelmann, 1999.

Lemma 4.6. Let $r: X \rightarrow Y$ be in SpecRel. Then the following assertions are equivalent.

(i) The relation $r$ is a partial map.

(ii) For each $x \in X$, the closed set $\{y \in Y \mid x r y\}$ is either empty or down-directed.

(iii) The diagram

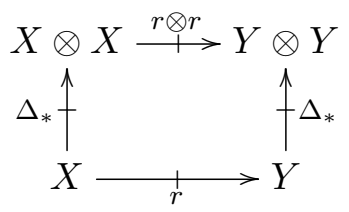


commutes in SpecRel.

Proof. Clearly (i) implies (ii), and (iii) is just a reformulation of (ii). To see (ii) $\Rightarrow(\mathrm{i})$, let $x \in X$ with $\{y \in Y \mid x r y\}$ down-directed. Then the closed set $\{y \in Y \mid x r y\}$ is irreducible and, since $Y$ is sober, is of the form $\overline{\left\{y_{0}\right\}}=\left\{y \in Y \mid y_{0} \leq y\right\}$ for some $y_{0} \in Y$.

We conclude that partial maps $r: X \mapsto Y$ in SpecRel correspond to binary infima preserving morphisms $f: A \rightarrow B$ in DLat $_{\perp, \vee}$.

\section{REFERENCES}

AdÁmek, J., Herrlich, H. and Strecker, G. E. (1990), Abstract and concrete categories: The joy of cats, Pure and Applied Mathematics (New York), John Wiley \& Sons Inc., New York, xiv+482 pages, Republished in: Reprints in Theory and Applications of Categories, No. 17 (2006) pp. 1-507, http://tac.mta.ca/tac/reprints/articles/17/tr17.pdf

Beck, J. M. (1967), Triples, algebras and cohomology, Ph.D. thesis, Columbia University, Republished in: Reprints in Theory and Applications of Categories, No. 2 (2003) pp 1-59, http://www.tac.mta. $\mathrm{ca} / \mathrm{tac} / \mathrm{reprints/articles/2/tr2 \cdot pdf}$.

Bonsangue, M. M., Kurz, A. and Rewitzky, I. M. (2007), Coalgebraic representations of distributive lattices with operators, Topology Appl. 154 (4), 778-791.

Celani, S. (2003), Generalized join-hemimorphisms on Boolean algebras, Int. J. Math. Math. Sci. (11), 681-693, http://eudml.org/doc/50418.

Cignoli, R., Lafalce, S. and Petrovich, A. (1991), Remarks on Priestley duality for distributive lattices, Order 8 (3), 299-315.

Clark, D. M. and Davey, B. A. (1998), Natural dualities for the working algebraist, volume 57 of Cambridge Studies in Advanced Mathematics, Cambridge University Press, Cambridge, xii+356 pages.

Clementino, M. M. and Tholen, W. (1997), A characterization of the Vietoris topology, in Proceedings of the 12th Summer Conference on General Topology and its Applications (North Bay, ON, 1997), volume 22, pages 71-95.

Dimov, G. D. and Tholen, W. (1989), A characterization of representable dualities, in Categorical topology and its relation to analysis, algebra and combinatorics (Prague, 1988), pages 336-357, World Sci. Publ., Teaneck, NJ.

Escardó, M. H. (1997), Injective spaces via the filter monad, in Proceedings of the 12th Summer Conference on General Topology and its Applications (North Bay, ON, 1997), volume 22, pages 97100.

Gierz, G., Hofmann, K. H., Keimel, K., Lawson, J. D., Mislove, M. W. and Scott, D. S. (1980), A compendium of continuous lattices, Springer-Verlag, Berlin, $\mathrm{xx}+371$ pages.

Halmos, P. R. (1956), Algebraic logic. I. Monadic Boolean algebras, Compositio Math. 12, 217-249.

Hofmann, D. (2014), The enriched Vietoris monad on representable spaces, accepted for publication in J. Pure Appl. Algebra arXiv:math.CT/1212.5539.

Johnstone, P. T. (1986), Stone spaces, volume 3 of Cambridge Studies in Advanced Mathematics, Cambridge University Press, Cambridge, xxii+370 pages, reprint of the 1982 edition.

Jónsson, B. and TARski, A. (1951), Boolean algebras with operators. I, Amer. J. Math. 73, 891-939.

Jónsson, B. and Tarski, A. (1952), Boolean algebras with operators. II, Amer. J. Math. 74, 127-162.

JUNG, A. (2004), Stably compact spaces and the probabilistic powerspace construction, in J. Desharnais and P. Panangaden, editors, Domain-theoretic Methods in Probabilistic Processes, volume 87, 15pp.

Kegelmann, M. (1999), Continuous domains in logical form, Ph.D. thesis, School of Computer Science, The University of Birmingham.

Kupke, C., Kurz, A. and Venema, Y. (2004), Stone coalgebras, Theoret. Comput. Sci. 327 (1-2), 109-134. 
Lawson, J. (2011), Stably compact spaces, Math. Structures Comput. Sci. 21 (1), 125-169.

MacDonald, J. and Sobral, M. (2004), Aspects of monads, in Categorical foundations, volume 97 of Encyclopedia Math. Appl., pages 213-268, Cambridge Univ. Press, Cambridge.

Manes, E. G. (1969), A triple theoretic construction of compact algebras, Sem. on Triples and Categorical Homology Theory, ETH Zürich 1966/67, Lect. Notes Math. 80, 91-118 (1969).

Nachbin, L. (1950), Topologia e Ordem, Univ. of Chicago Press, in Portuguese, English translation: Topology and Order, Van Nostrand, Princeton (1965).

Negrepontis, J. W. (1971), Duality in analysis from the point of view of triples, J. Algebra 19, 228-253.

Petrovich, A. (1996), Distributive lattices with an operator, Studia Logica 56 (1-2), 205-224, special issue on Priestley duality.

Poppe, H. (1966), Einige Bemerkungen über den Raum der abgeschlossenen Mengen, Fund. Math. 59, 159-169.

Porst, H.-E. (1994), What is concrete equivalence?, Appl. Categ. Structures 2 (1), 57-70.

Porst, H.-E. and Tholen, W. (1991), Concrete dualities, in Category theory at work (Bremen, 1990), volume 18 of Res. Exp. Math., pages 111-136, Heldermann, Berlin.

Priestley, H. A. (1970), Representation of distributive lattices by means of ordered stone spaces, Bull. London Math. Soc. 2, 186-190.

Priestley, H. A. (1972), Ordered topological spaces and the representation of distributive lattices, Proc. London Math. Soc. (3) 24, 507-530.

Pumplün, D. (1970), Eine Bemerkung über Monaden und adjungierte Funktoren, Math. Ann. 185, $329-337$.

Pumplün, D. (1988), Eilenberg-Moore algebras revisited, Seminarberichte, FB Mathematik u. Inf., Fernuniversität Hagen 29, 97-144.

Sambin, G. and Vaccaro, V. (1988), Topology and duality in modal logic, Ann. Pure Appl. Logic 37 (3), 249-296.

Schalk, A. (1993), Algebras for Generalized Power Constructions, Ph.D. thesis, Technische Hochschule Darmstadt.

Simmons, H. (1982), A couple of triples, Topology Appl. 13 (2), 201-223.

Stone, M. H. (1936), The theory of representations for Boolean algebras, Trans. Amer. Math. Soc. 40 (1), 37-111.

Stone, M. H. (1938), Topological representations of distributive lattices and Brouwerian logics, Časopis pro pěstování matematiky a fysiky 67 (1), 1-25, http://dml.cz/handle/10338.dmlcz/124080.

Stralka, A. (1980), A partially ordered space which is not a Priestley space, Semigroup Forum 20 (4), 293-297.

Tholen, W. (1974), Relative Bildzerlegungen und algebraische Kategorien, Ph.D. thesis, Westfälische Wilhelms-Universität Münster.

Tholen, W. (2009), Ordered topological structures, Topology Appl. 156 (12), 2148-2157.

Vietoris, L. (1922), Bereiche zweiter Ordnung, Monatsh. Math. Phys. 32 (1), 258-280.

Wright, F. B. (1957), Some remarks on Boolean duality, Portugal. Math. 16, 109-117, http://eudml. org/doc/114792.

Center for Research and Development in Mathematics and Applications, Department of Mathematics, University of Aveiro, 3810-193 Aveiro, Portugal

E-mail address: dirk@ua.pt

E-mail address: a28224@ua.pt 